\title{
Identification of dysregulated microRNAs associated with diagnosis and prognosis in triple-negative breast cancer: An in silico study
}

\author{
CHUNNI FAN and NING LIU \\ Department of Breast Surgery, China-Japan Union Hospital of Jilin University, Changchun, Jilin 130033, P.R. China
}

Received September 5, 2018; Accepted February 4, 2019

DOI: $10.3892 /$ or.2019.7094

\begin{abstract}
Triple-negative breast cancer (TNBC) is a highly aggressive subtype of breast cancer with limited treatment options combined with poor rates of survival. Given the lack of appropriate prognostic biomarkers for TNBC patients, the present study aimed to identify potential dysregulated miRNAs capable of providing a diagnosis and predicting overall survival for TNBC patients. A total of 289 miRNAs were aberrantly regulated in TNBC tissue compared to adjacent, non-cancerous tissues and 96 microRNAs (miRNAs) in TNBC compared with non-triple-negative breast cancer (nTNBC) samples. Receiver operating characteristic (ROC) curve analysis suggested that 4 miRNAs (hsa-miR-10a, hsa-miR-18a, hsa-miR-135b and hsa-miR-577) had diagnostic value [area under curve (AUC) $>0.8$ ]. A 4-miRNA signature consisting of hsa-miR-148b, hsa-miR-203a, hsa-miR-203b and hsa-miR-3922 was constructed for prediction of prognosis. A multivariate Cox's proportional hazards regression model indicated that the 4-miRNA signature was an independent prognostic factor of other clinical variables in patients with TNBC. Functional analysis of the target genes of the miRNA signature demonstrated that the prolactin signaling pathway and miRNAs in cancer were significantly enriched. In conclusion, the results in the present study may highlight efficient biomarkers for the diagnosis of TNBC and its prognosis. In-depth exploitation of these miRNAs will help define and develop novel molecular therapeutic strategies and improve prognosis for TNBC patients.
\end{abstract}

Correspondence to: Dr Ning Liu, Department of Breast Surgery, China-Japan Union Hospital of Jilin University, 126 Xiantai Street, Changchun, Jilin 130033, P.R. China

E-mail: liuning120324@163.com

Abbreviations: TNBC, triple-negative breast cancer; ROC, receiver operating characteristic; TCGA, The Cancer Genome Atlas; AUC, area under curve; miRNA, microRNA

Key words: triple-negative breast cancer, miRNA signature, overall survival, prognosis, Cox's proportional hazards regression model

\section{Introduction}

Globally, breast cancer remains the leading cause of cancerrelated deaths in females. An estimated 1.7 million new cases were diagnosed and 521,900 breast cancer patients succumbed to this disease in 2012 (1). Similarly, in China, a diagnosis of breast cancer between the ages of 30 and 59 years is more common than for any other type of cancer except that of the thyroid (2). Breast cancer is classified into five intrinsic subtypes through detection of progesterone receptor (PR), estrogen receptor-alpha $(\mathrm{ER} \alpha)$ and human epidermal growth factor receptor 2 (HER2), namely luminal A-like $\left(E R \alpha^{+}\right.$ and/or $\mathrm{PR}^{+}$and HER2-), luminal B-like (ER $\alpha^{+}$and/or $\mathrm{PR}^{+}$ and HER2 $2^{+}$), HER2 overexpression (ER $\alpha^{-}, \mathrm{PR}^{-}$and HER2 $\left.{ }^{+}\right)$ and triple-negative breast cancer (TNBC) (HER2-, ER $\alpha^{-}$and PR') and normal-like tumors (3). Luminal A-like tumors have higher expression of ER-related genes and lower expression of proliferative genes than luminal B-like cancers (4). Growing evidence indicates that TNBC is a highly aggressive tumor with limited treatment strategies and has poorer survival outcomes compared with other subtypes of breast cancer. Although TNBC patients do benefit from chemotherapy, more effective and less toxic treatments are required in order to reduce the risk of disease progression and improve the prognosis. Therefore, further studies are urgently needed to screen molecular biomarkers to determine the therapeutic efficacy of treatments, and improve the performance of diagnosis and prognosis of TNBC.

MicroRNAs (miRNAs) are small 20-22 nucleotide noncoding RNAs that are known to regulate the expression of genes participating in the control of cell proliferation, apoptosis, development and stress response by binding to the $3^{\prime}$ or $5^{\prime}$ untranslated region of target transcripts (5). Furthermore, miRNAs are also involved in tumorigenesis by acting as either tumor suppressors or oncogenes. This suggests that miRNAs can potentially be biomarkers for cancer diagnosis and prognosis. It has been reported that four miRNAs, namely hsa-miR-125b, hsa-miR-16, hsa-miR-155 and hsa-miR-374a are significantly associated with overall survival of TNBC patients, of which three are correlated with better prognosis and one with worse prognosis (6). In addition, a 4-miRNA signature defined by the expression levels of miR-155, miR-493, miR-30e and miR-27a has both diagnostic and prognostic value for predicting outcomes of TNBC patients 
most commonly treated with chemotherapy (7). Furthermore, another 4-miRNA signature (miR-18b, miR-103, miR-107 and miR-652) may accurately predict tumor relapse and overall survival of TNBC patients (8). Although several miRNA signatures have been identified which could be used for TNBC diagnosis and survival prediction, the novel prognosis strategy used by miRNA signatures has not been applied in clinical studies. Hence, it is useful to screen new miRNA biomarkers for TNBC overall survival.

In the present study, in order to identify diagnostic and prognostic miRNAs in TNBC patients, we analyzed large scale clinical data and miRNA sequencing data from The Cancer Genome Atlas (TCGA) datasets. After identification of differentially expressed miRNAs in TNBC, miRNAs with diagnostic and prognostic value were identified and a 4-miRNA signature was determined and then used to predict overall survival.

\section{Materials and methods}

Retrieval of breast cancer clinical and miRNA expression data. A total of 1,098 anonymized patients were identified in the TCGA database as having breast cancer. The clinical data were retrieved from the TCGA data portal (https://tcga-data. nci.nih.gov/tcga/version 10.0, release time: December 21, 2017; Species: human) on January 22, 2018. Of these 1,098 patients, 155 were diagnosed as having TNBC based on their ER, PR and Her-2 status and defined using immunohistochemistry (IHC). Three patients who were diagnosed as TNBC did not have miRNA expression data recorded and thus were excluded. The other 943 patients were diagnosed as non-triple-negative breast cancer (nTNBC) subtypes (positive expression of ER, PR or Her-2). Finally, a total of 152 TNBC and 943 nTNBC patients were included in the present study. The detailed clinic data is presented in Table I. The follow-up time was different for every patient and the longest time was 3,472 days.

In addition, 1,207 miRNA sequencing datasets from frozen tumor samples by Illumina HiSeq 2000 platform (Illumina, Inc., San Diego, CA, USA) were also downloaded from the TCGA data portal. Of these, 1,103 were tumor samples and 104 were associated with adjacent normal tissue. A total of 200 had unknown ER, PR or Her-2 status and were thus excluded. In total, the miRNA sequencing data from $152 \mathrm{TNBC}$ and $751 \mathrm{nTNBC}$ samples were used for dysregulated miRNA exploration.

Identification of dysregulated miRNAs from TNBC patients. To discover differentially expressed miRNAs from TNBC patients, the edgeR software package of the Bioconductor project (9) using the $\mathrm{R}$ programming environment with default parameter settings was utilized. miRNAs from TNBC samples were considered dysregulated in comparison with adjacent normal samples and nTNBC tissue. Differences were assessed with the Mann-Whitney $U$ test and were significant if $\log \mathrm{FC}$ (fold change) $\mid>1$ and $\mathrm{P}<0.05$. Furthermore, gplots (version 3.0.1; https://cran.r-project.org/src/contrib/Archive/ gplots/) and pheatmap (version 1.0.8; https://CRAN.R-project.org/package $=$ pheatmap) packages were used to study the expression levels and distribution of miRNAs expressed
Table I. Clinical features of all 152 TNBC patients included in the present study.

\begin{tabular}{lc}
\hline Features & $\mathrm{N}(\%)$ \\
\hline Age (years) & \\
$<60$ & $100(65.79)$ \\
$\geq 60$ & $52(34.21)$ \\
Sex & \\
Female & $152(100)$ \\
Male & $0(0)$ \\
Vital status & \\
Alive & $134(88.16)$ \\
Dead & $18(11.84)$ \\
Pathological stage & \\
I & $29(19.08)$ \\
II & $97(63.82)$ \\
II & $24(15.79)$ \\
IV & $2(0.01)$ \\
Tumor size & \\
T1 & $41(26.97)$ \\
T2 & $92(60.53)$ \\
T3 & $15(9.87)$ \\
T4 & $4(2.63)$ \\
Lymph node & \\
N0 & $102(67.10)$ \\
NX & $50(32.90)$ \\
Metastasis status & \\
M0 & $129(84.87)$ \\
MX & $23(15.13)$ \\
\hline NX in &
\end{tabular}

$\mathrm{NX}$, including patients at stage N1-N3; MX, including two patients at stage M1, others at MX were not determined with metastasis; TNBC, triple-negative breast cancer.

differentially between the TNBC and the normal samples as well as the nTNBC tissue with default parameter settings.

Identification of miRNAs with diagnostic value. To identify differentially dysregulated miRNAs that had capacity for diagnosing TNBC, receiver operating characteristic (ROC) curves were plotted to compute the sensitivity and specificity of each miRNA associated with TNBC diagnosis using the pROC software package (version 1.10.0; http://cran.r-project. org/web/packages/pROC/) with default parameter settings. Sensitivity was defined as the percent of tumor cases with a diagnostic test exceeding a criterion and specificity as the percent of non-tumor cases less than or equal to that criterion with a diagnostic test. Dysregulated miRNAs with an area under curve (AUC) $>0.8$ were selected and miRNAs common to two comparison groups were considered as having diagnostic value.

Survival analysis. The entire set including 104 normal and 152 TNBC samples were randomly divided into a training 

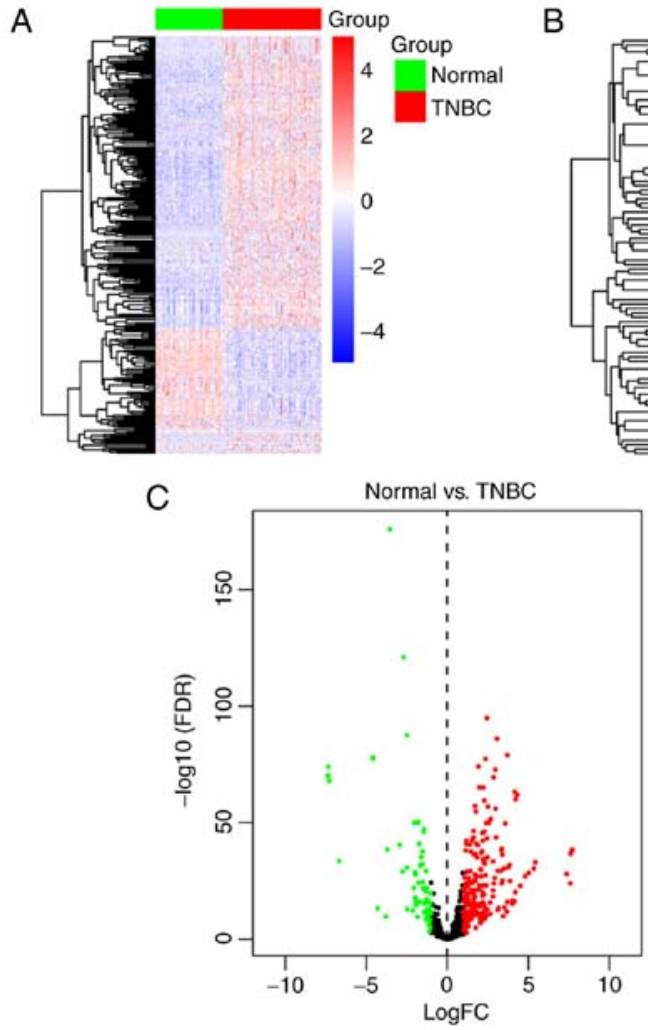
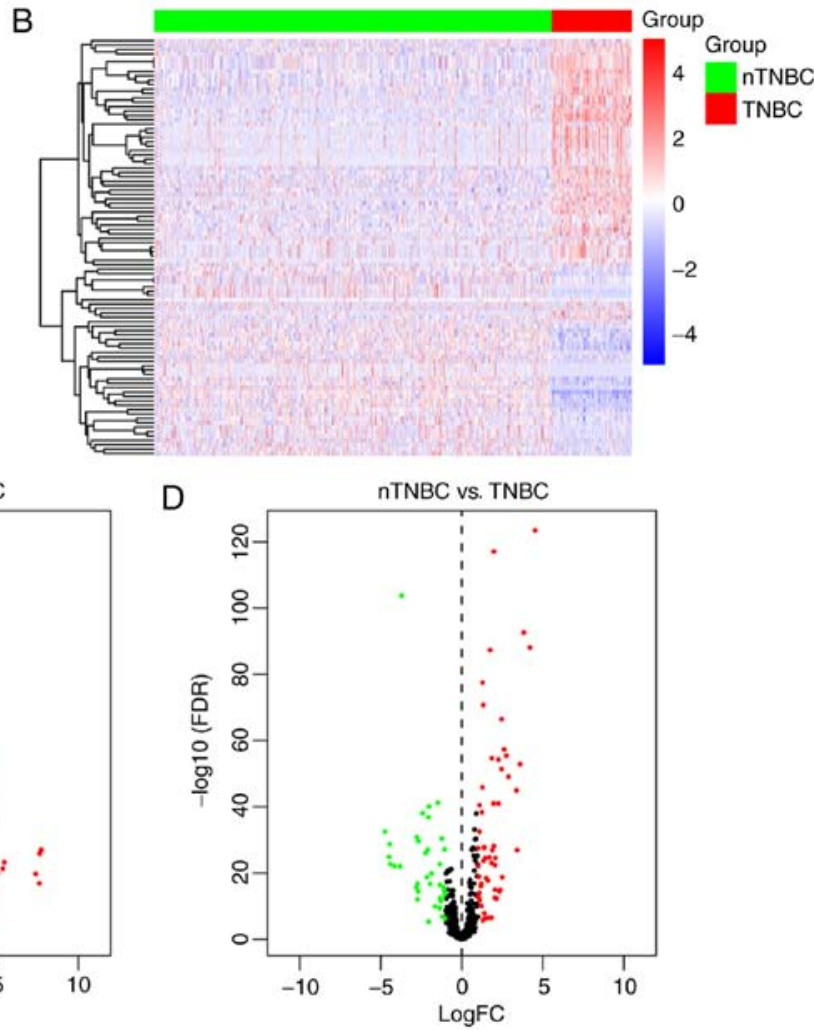

Figure 1. Dysregulated miRNAs in TNBC compared with normal samples and nTNBC. (A and B) Expression heatmap and (C and D) volcano plot of miRNAs in comparison of normal vs. TNBC and nTNBC vs. TNBC. TNBC, triple-negative breast cancer; nTNBC, non-triple-negative breast cancer; FC, fold change; FDR, false discovery rate.

set (51 normal and 77 TNBC samples) and a validation set (53 normal and 75 TNBC samples). To evaluate the relationship between the miRNA expression levels and the overall survival in tumor patients, univariate Cox regression analysis was conducted with the aim of identifying potential miRNAs related to TNBC prognosis. miRNAs that were clearly associated with patient survival were included when $\mathrm{P}<0.05$ and then subjected to stepwise multivariate Cox regression analysis to construct a TNBC prognostic signature based on the following formula:

$$
\text { Risk score }=\exp _{1} \times \beta_{1}+\exp _{2} \times \beta_{2}+\ldots+\exp _{n} \times \beta_{n}
$$

where $n$ was the number of the prognostic miRNA, $\beta$ was the regression coefficient and exp was the expression level of that miRNA. After risk score acquisition of each patient, patients in the training set were divided into the high-risk and the low-risk groups using the median score as the central cut-off point. In addition, univariate and multivariate Cox proportional hazards regression analyses were performed to examine the relationship between the risk score of TNBC patients and other clinical features including age $(<60$ or $\geq 60)$, pathologic stage (I-II or III-IV), stage T, stage N and stage $\mathrm{M}$. ROC curves were plotted and AUC values were calculated. Patient survival was evaluated using the Kaplan-Meier method and log-rank tests using R package 'survival' (version: 2.42-3; https://CRAN.R-project.org/package=survival) with default parameter settings. A 95\% confidence interval (CI) and hazard ratio (HR) were calculated to evaluate the prognostic variables related to TNBC survival.
Target prediction of potential miRNA signatures and functional annotation. To gain more insight into the role of prognostic miRNA signatures in TNBC, miRNA-target prediction programs were used to predict the target genes of the miRNAs, including miRDB (http://www.miRdb.org/ index.html), miRTarBase (http://miRtarbase.mbc.nctu.edu. tw/php/index.php) and TargetScan (http://www.targetscan. org/vert_71/). Genes that were commonly identified by the three tools were considered as target mRNAs of a prognostic miRNA signature. To reveal the potential roles of the target genes, clusterProfileR package (version 3.6) in the R environment (10) with default parameter settings was employed to perform Gene Ontology (GO) and Kyoto Encyclopedia of Genes and Genomes (KEGG) enrichment analyses. $\mathrm{P}<0.05$ was set as the cut-off.

\section{Results}

Identification of differentially expressed miRNAs in TNBC and $n T N B C$ patients. To identify dysregulated miRNAs in TNBC patients in comparison with adjacent normal tissue and miRNAs in TNBC compared with nTNBC samples, the edgeR package was utilized, with 216 upregulated and 73 downregulated miRNAs based on the cut-off of $\mathrm{P}<0.05$ and $\| \log \mathrm{FCl}>1$ in TNBC compared with adjacent normal samples. When compared with nTNBC samples, there were 58 upregulated and 38 downregulated miRNAs in tumor samples from TNBC patients. Hierarchical clustering of differentially expressed miRNAs presented clear separation in the expression profiles of TNBC compared with normal samples (Fig. 1A) 

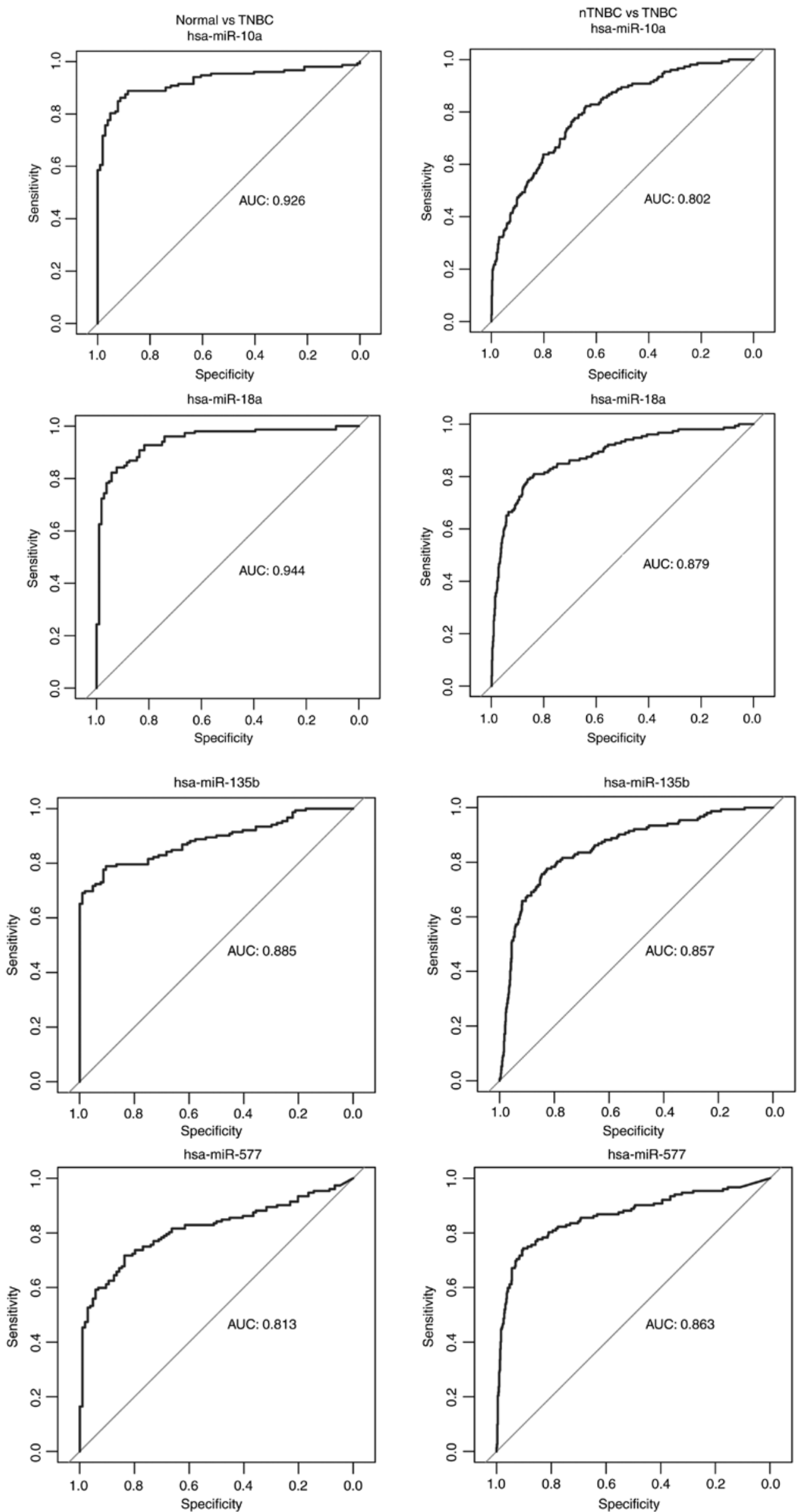

Figure 2. ROC curves of the 4 miRNAs with diagnostic value in the two comparison groups. The AUC was $>0.8$. ROC, receiver operating characteristic; AUC, area under curve; TNBC, triple-negative breast cancer; nTNBC, non-triple-negative breast cancer. 



Figure 3. Expression of 4 diagnostic miRNAs in the two comparison groups. The plots demonstrate that the expression levels of the 4 miRNAs in TNBC samples were different from that in the normal or nTNBC tissues. TNBC, triple-negative breast cancer; nTNBC, non-triple-negative breast cancer. ${ }^{* * * * *} \mathrm{P}<0.001$.

and nTNBC patients (Fig. 1B). A volcano plot was created to indicate the dysregulated expressed miRNAs (Fig. 1C and D).

Diagnostic value of dysregulated miRNAs. ROC analyses were performed to evaluate the possible diagnostic capacity of each dysregulated miRNA. Differentially expressed miRNAs with an AUC $>0.8$ were selected as miRNAs likely to be useful as biomarkers in the diagnosis of TNBC. We obtained 27 miRNAs from the comparison between the TNBC and the adjacent normal tissue and 6 miRNAs from the comparison between the TNBC and the nTNBC samples with an AUC $>0.8$. There were 4 common miRNAs between two comparisons, namely hsa-miR-10a, hsa-miR-18a, hsa-miR-135b and hsa-miR-577 (Fig. 2). The expression levels of these 4 miRNAs in TNBC, adjacent normal samples and nTNBC are presented in Fig. 3. Compared with the adjacent normal breast tissues and nTNBC, the expression of hsa-miR-10a was lower in the TNBC tumors however the expression of the other three miRNAs was higher $\left(\log _{2} \mathrm{FC}>1 \mathrm{l}, \mathrm{P}<0.001\right)$. The results indicated that these miRNAs were specifically upregulated and downregulated in TNBC and could be used as diagnostic biomarkers for the diagnosis of TNBC.

Identification of miRNAs associated with TNBC prognosis. Kaplan-Meier plots and log-rank tests were used to identify miRNAs related to overall survival of TNBC patients with a cut-off log-rank (LR) $\mathrm{P}<0.05$. A total of 8 miRNAs were associated with overall survival, namely: hsa-miR-147b, hsa-miR-203a, hsa-miR-377, hsa-miR-429, hsa-miR-511, hsa-miR-1247, hsa-miR-3922 and hsa-miR-7706 (Fig. 4A). ROC analysis was then performed to assess the prognostic capacity of the miRNAs to predict survival. The AUCs of 
A
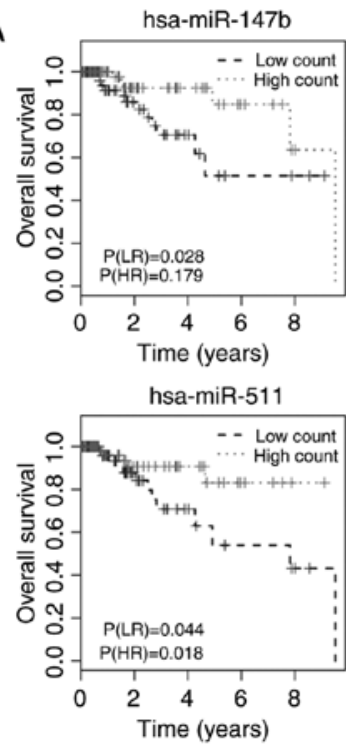

B

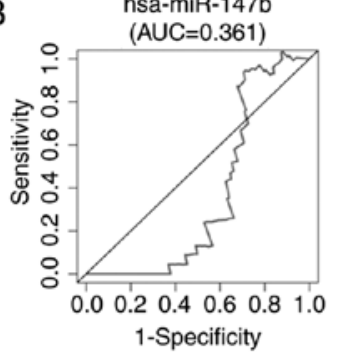

hsa-miR-511

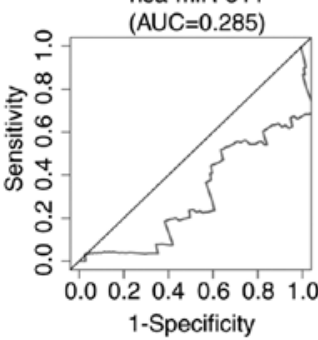

hsa-miR-203a

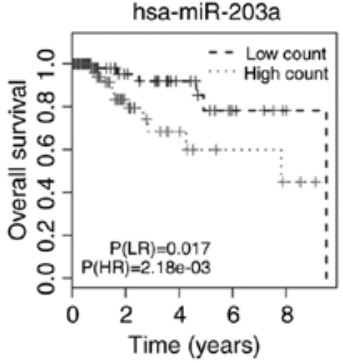

hsa-miR-1247

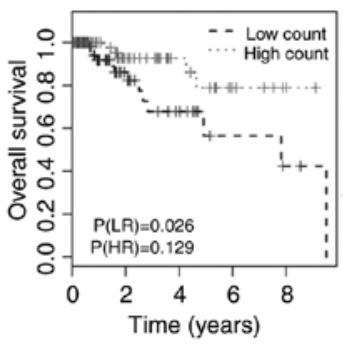

hsa-miR-203a

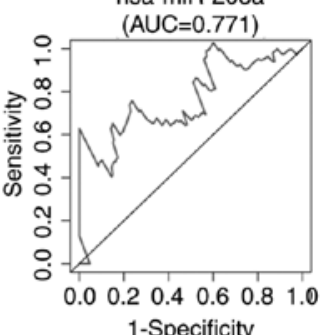

hsa-miR-1247

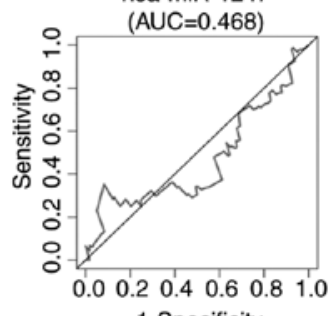

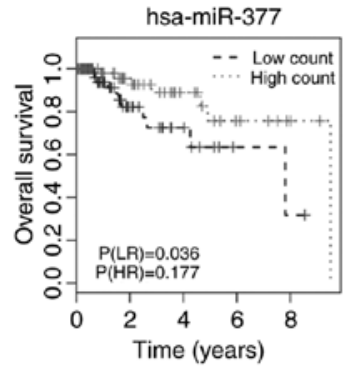

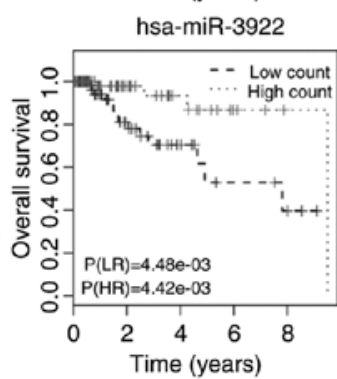

hsa-miR-377

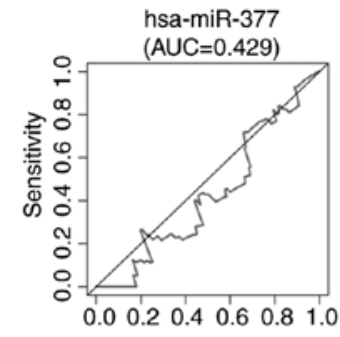

1-Specificity

hsa-miR-3922

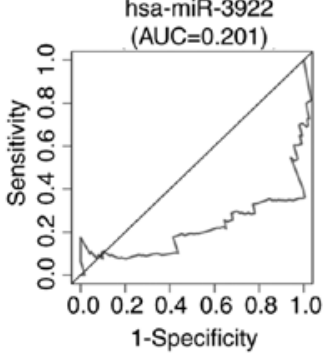

hsa-miR-429
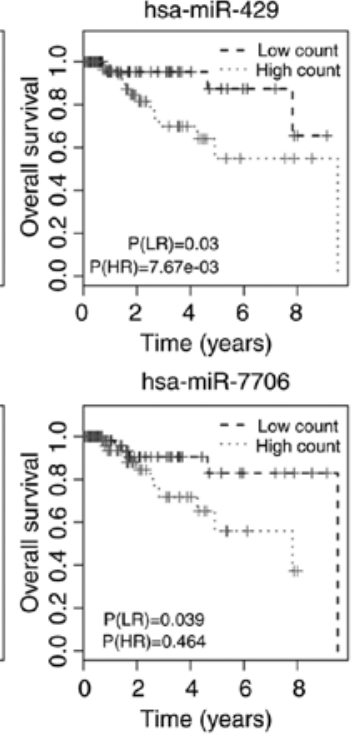

hsa-miR-429

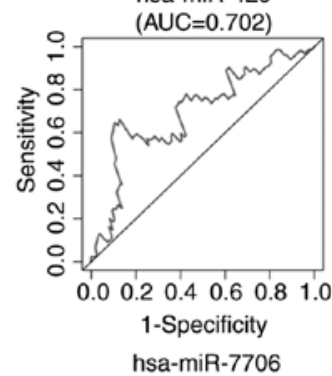

(AUC=0.624)

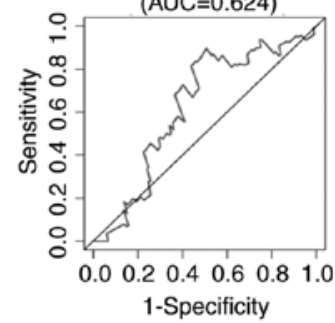

Figure 4. Kaplan-Meier and ROC curves for miRNAs associated with overall survival of TNBC patients. (A) The differences between the low- and high-count groups were determined using an LR test. (B) The AUC values are presented with each ROC curve. ROC, receiver operating characteristic; TNBC, triple-negative breast cancer; nTNBC, non-triple-negative breast cancer; LR, log-rank; AUC, area under curve; HR, hazard ratio.

the 8 miRNAs are displayed in Fig. 4B, and indicated that 2 miRNAs, hsa-miR-203a and hsa-miR-429, may have prognostic value. The AUCs for the 2 miRNAs predicting 5 -year survival were 0.771 and 0.702 , respectively.

The predictive capacity of a 4-miRNA signature in TNBC. A univariate Cox's proportional hazards regression model was fitted to the entire set and 5 miRNAs (hsa-miR-148b, hsa-miR-203a, hsa-miR-203b, hsa-miR-3922 and hsa-miR-429) were identified as being associated with overall survival with $\mathrm{P}(\mathrm{HR})<0.01$. A group of miRNAs that could be defined as a signature, namely hsa-miR-148b, hsa-miR-203a, hsa-miR-203b and hsa-miR-3922 was identified after stepwise multivariate Cox's regression model analysis by calculating the prognostic risk score. TNBC patients were divided into a low- or a high-risk group depending on their score relative to that of the median risk score (Fig. 5A). The mortality rate of the high-risk group was $19.74 \%$ and that of the low-risk group was $3.95 \%$, the difference being significant $(\mathrm{P}<0.05$;
Fig. 5B). The heatmap presented in Fig. 5C demonstrated that the 4-miRNA signature was expressed differently in the low- and the high-risk groups. The expression pattern of the 4-miRNA signature in TNBC and normal samples, and the low- and the high-risk groups are presented in Fig. 6. These 4 miRNAs were significantly upregulated in TNBC patients compared with the normal controls $(\mathrm{P}<0.001)$. Similarly, three miRNAs, hsa-miR-148b, hsa-miR-203a and hsa-miR-203b, were expressed at a higher level in the high-risk patients than in samples from the low-risk group $(\mathrm{P}<0.001)$. However, the expression of hsa-miR-3922 showed the opposite expression pattern $(\mathrm{P}<0.001)$.

The 4-miRNA signature is an independent prognostic factor associated with overall survival. Finally, both univariate and multivariate Cox's proportional hazards regression model analyses were performed to evaluate the prognostic power of the 4-miRNA signature. The univariate Cox's regression model demonstrated that pathological stage, $\mathrm{N}$ stage, $\mathrm{T}$ stage and 
A

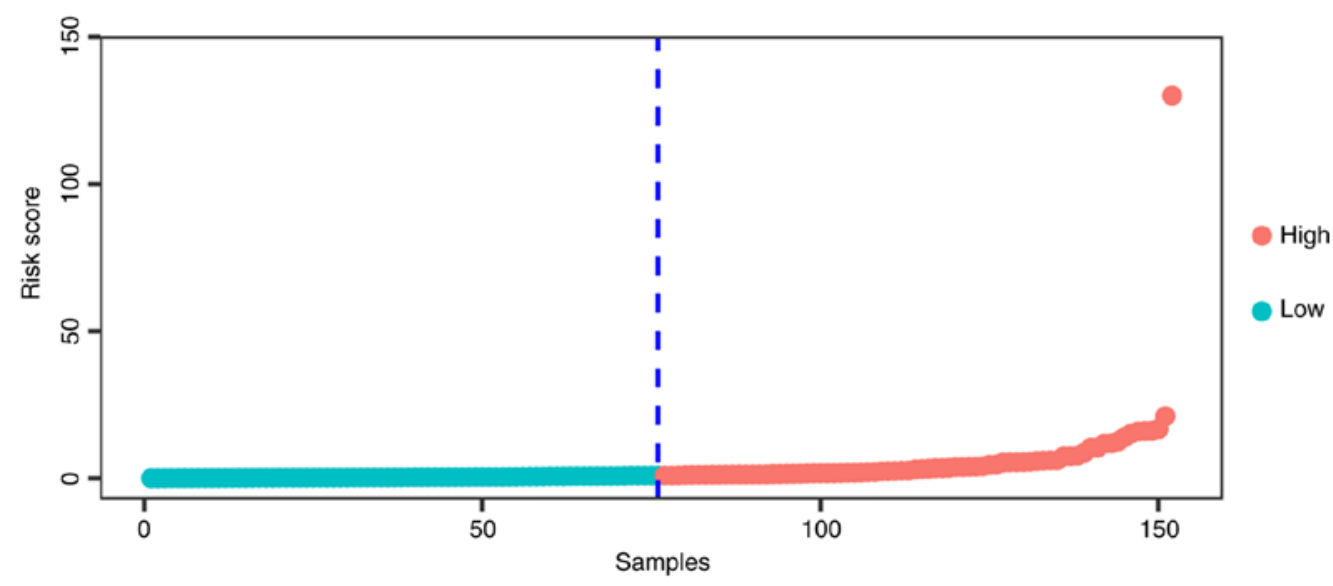

B



C

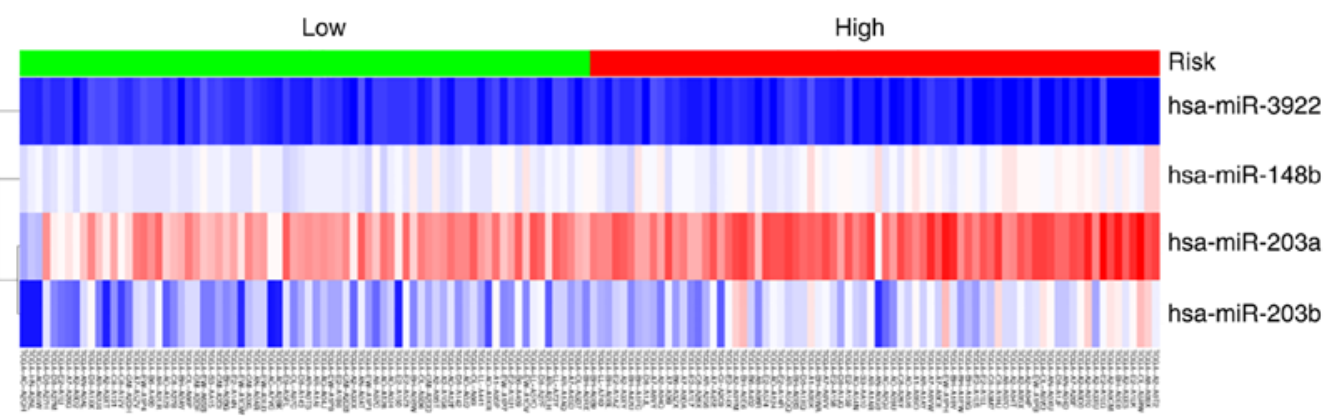

Figure 5. The 4-miRNA signature is associated with the overall survival of TNBC patients. (A) Patients were divided into the low- and the high-risk groups at the median risk score point. The x-axis indicates the number of patients. (B) Survival time of all TNBC patients. The x-axis indicates the number of patients. (C) The expression of the 4-miRNA signature in the low- and the high-risk groups. The $\mathrm{x}$-axis shows the patients in TCGA database. TNBC, triple-negative breast cancer.

Risk score based on the 4-miRNA signature were significantly correlated with overall survival of TNBC patients $(\mathrm{P}<0.05)$, while the multivariate Cox's regression model revealed that only the pathological stage and Risk score were independent prognostic factors associated with overall survival $(\mathrm{P}=0.002$ and 0.021, respectively; Table II). Kaplan-Meier curves of the clinical characteristics and risk score are displayed in Fig. 7A. The highest survival rate was found within the low-risk group in comparison with the high-risk group $(\mathrm{P}=0.0065)$. The AUC for the 4-miRNA signature predicting 5-year survival of TNBC patients was 0.902 (Fig. 7B). Our results suggest that the 4-miRNA signature may have prognostic value for predicting the overall survival of TNBC patients.
Functional annotation of the target genes of the miRNA signature. After target prediction of the 4 miRNAs (hsa-miR-148b, hsa-miR-203a, hsa-miR-203b and hsa-miR-3922) using miRDB, miRTarBase and TargetScan, GO function and KEGG pathway enrichment analysis of the target genes was performed using the $\mathrm{R}$ clusterProfiler software package. Details of target genes of the 4 miRNAs are presented in Table III. According to the results of the GO functional enrichment analysis, regulation of anoikis, negative regulation of cellular amide metabolic process and regulation of protein insertion into mitochondrial membranes involved in apoptotic signaling were the most significantly enriched biological processes. The most clearly enriched cellular 
Table II. The predictive values of clinical features and risk score.

\begin{tabular}{|c|c|c|c|c|c|}
\hline \multirow[b]{2}{*}{ Variables } & \multirow[b]{2}{*}{ Patients $(\mathrm{N})$} & \multicolumn{2}{|c|}{ Univariate analysis } & \multicolumn{2}{|c|}{ Multivariate analysis } \\
\hline & & $\operatorname{HR}(95 \% \mathrm{CI})$ & P-value & $\mathrm{HR}(95 \% \mathrm{CI})$ & P-value \\
\hline \multicolumn{6}{|l|}{ Age (years) } \\
\hline$<60 / \geq 60$ & $100 / 52$ & $0.95(2.7-0.33)$ & 0.920 & $0.5(0.15-1.67)$ & 0.259 \\
\hline \multicolumn{6}{|c|}{ Pathological stage } \\
\hline I-II/III-IV & $126 / 26$ & $22.21(81.6-6.05)$ & 0.000 & $16.56(2.79-98.38)$ & 0.002 \\
\hline \multicolumn{6}{|l|}{ Stage T } \\
\hline $\mathrm{T} 1-\mathrm{T} 2 / \mathrm{T} 3-\mathrm{T} 4$ & $133 / 19$ & $6.99(22.99-2.13)$ & 0.001 & $2.28(0.58-8.98)$ & 0.239 \\
\hline \multicolumn{6}{|l|}{ Stage N } \\
\hline N0/NX & $102 / 50$ & $5.43(16.67-1.77)$ & 0.003 & $2.51(0.51-12.46)$ & 0.259 \\
\hline \multicolumn{6}{|l|}{ Stage M } \\
\hline M0/MX & $129 / 23$ & $0.57(4.39-0.07)$ & 0.589 & $0.12(0.01-1.27)$ & 0.079 \\
\hline \multicolumn{6}{|l|}{ Risk score } \\
\hline Low/high & $76 / 76$ & $9.32(41.07-2.11)$ & 0.003 & $6.75(1.33-34.21)$ & 0.021 \\
\hline
\end{tabular}
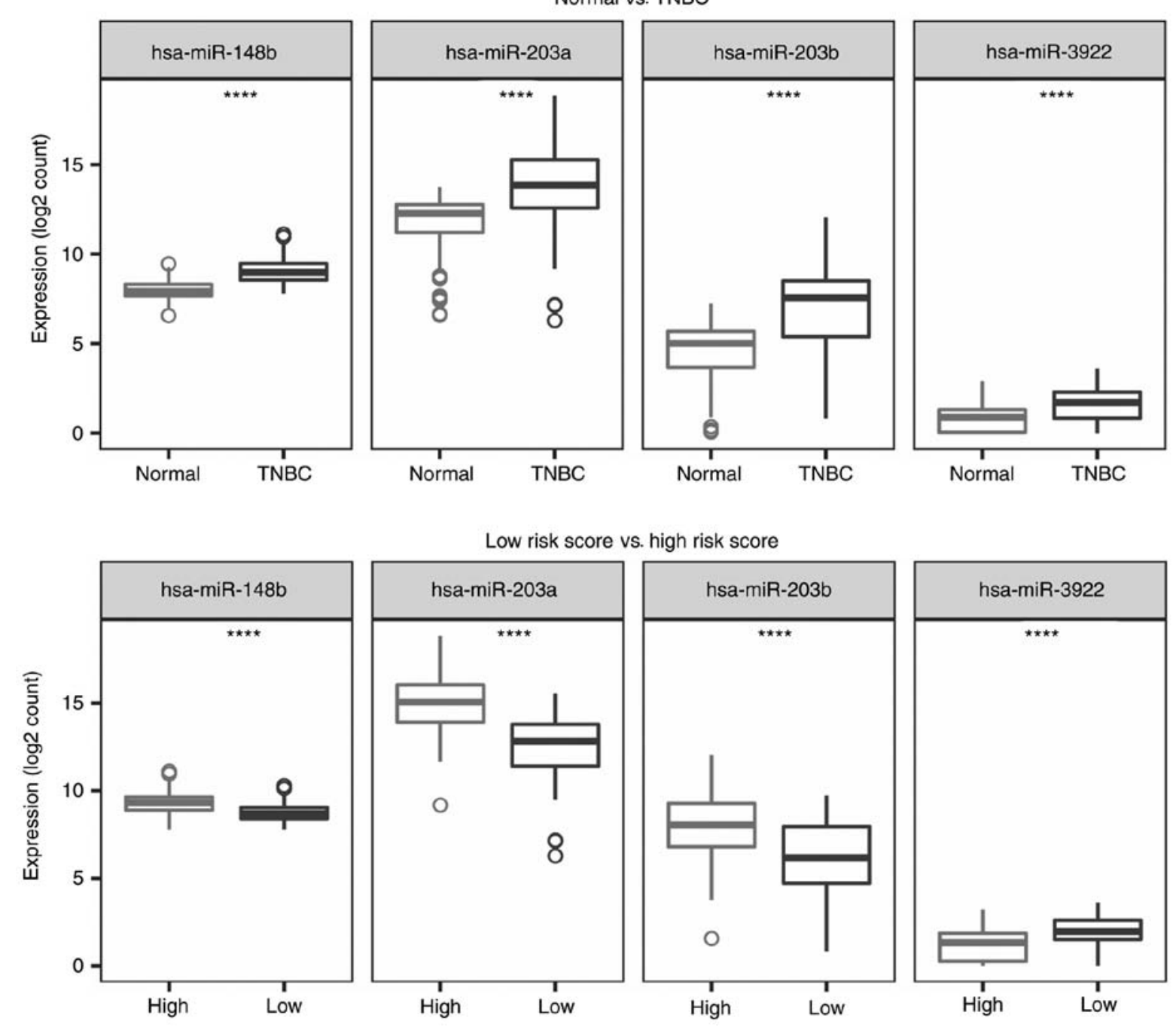

Figure 6. Expression levels of the 4-miRNA signature in comparison of TNBC with normal tissue and comparison of the low- and the high-risk groups. ${ }_{* * * * * *} \mathrm{P}<0.001$. TNBC, triple-negative breast cancer. 
Table III. miRNAs targeting mRNAs of TNBC.

miRNA

mRNA

hsa-mir-148b SYNCRIP, TNRC6A, WASL, MLEC, BTBD3, YWHAB, PPP6R1, USP33, NPTX1, CUL5, C1GALT1, AGO1, SECISBP2L, MTMR10, ATP6AP2, ZCCHC2, DNMT1, PRKAA1, DICER1, RTN4, CCT6A, ZFYVE26, ARL8B, DLG2, ATP7A, SESTD1, ACVR1, ALCAM, OTUD4, FBXO28, ITSN2, KLF6, CDK19, NPEPL1, CLCN3, MAP3K9, CYB5R4, ZDHHC17, EOGT, SIK1, RAB14, PAPD4, TBL1XR1, RAB34, GLRX5, CEP55, NRAS, NCKIPSD, FAM104A, SOS2, C3orf58, PRNP, DCP2, STARD13, OSBPL11, DDX6, FLOT2, ABCB7, BMP3, MARCH2, RAB12, JARID2, USP48, AP4E1, ITGA5, TXNIP, NSD1

hsa-mir-203a TFAM, SHOC2, ERI2, SOCS5, GAN, CACNB2, BTG2, TSC22D2, YWHAE

hsa-mir-203b SPTY2D1, VEZF1, EFHD2, TIPARP

TNBC, triple-negative breast cancer.


B

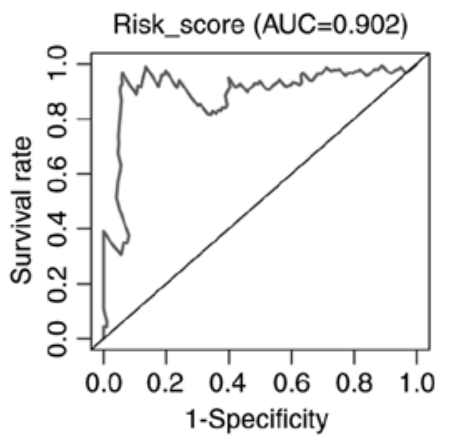

Figure 7. (A) Kaplan-Meier plots for various stages of disease or clinical characteristics and risk score based on the 4-miRNA signature. (B) ROC curve of risk score based on the 4-miRNA signature. ROC, receiver operating characteristic; AUC, area under curve.

components were RNAi effector complex, ribonucleoprotein granule, RNA-induced silencing complex (RISC) and ribonucleoprotein granule. The most significantly enriched signaling pathways determined through KEGG analysis were the prolactin signaling pathway, the FOXO signaling pathway, and the ErbB and Insulin signaling pathways, and miRNAs and choline metabolism in cancer. The results of enrichment analysis are displayed in Fig. 8. 


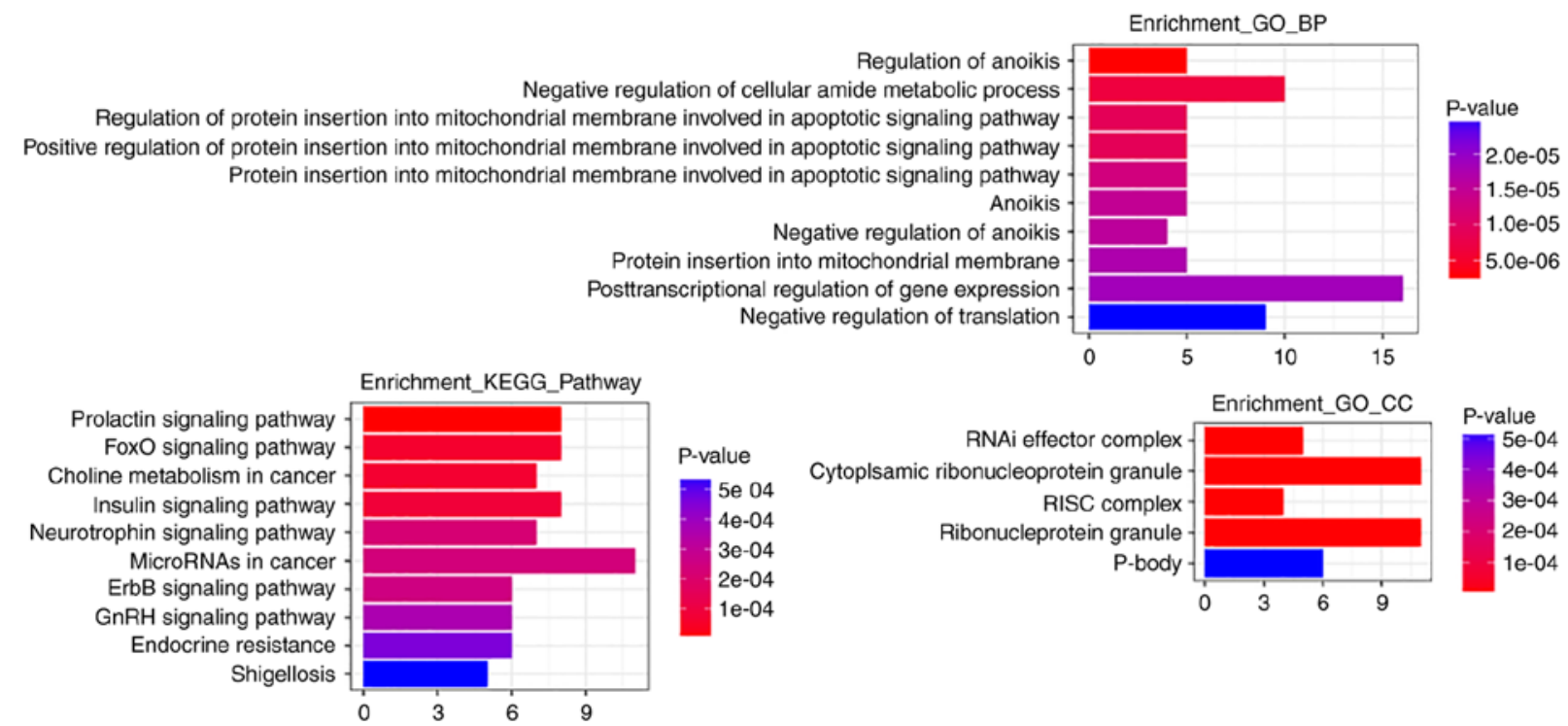

Figure 8. Functional annotation and pathway enrichment analysis of targets for the 4-miRNA signature from GO (April 5, 2018) and KEGG (Release 86.0, April 1, 2018) annotation databases. GO, Gene Ontology; BP, biological process; CC, cellular component; KEGG, Kyoto Encyclopedia of Genes and Genomes.

\section{Discussion}

Growing evidence indicates that triple-negative breast cancer (TNBC) is a heterogeneous disease comprising several distinct disorders with clearly different clinical behavior and molecular characteristics $(11,12)$. However, no specific and well-defined molecular targets have thus far been defined in TNBCs, and therefore few therapeutic strategies can be utilized as treatments, or are on the development horizon. In the present study, we found 4 miRNAs (hsa-miR-10a, hsa-miR-18a, hsa-miR-135b and hsa-miR-577) with significant value in TNBC diagnosis. We conducted a detailed analysis of a 4-miRNA signature which was comprised of hsa-miR-148b, hsa-miR-203a, hsa-miR-203b and hsa-miR-3922 and exhibited capacity for predicting TNBC overall survival.

In previous studies, hsa-miR-10a belonging to the miR-10 family has been revealed to be dysregulated in several types of cancers (13), such as breast (14), glioblastoma (15), lung cancer (16) and chronic lymphocytic leukemia (17). Another member of the miR-10 family, miR-10b, has been demonstrated to functionally contribute to tumor invasion and metastasis in breast cancer (18). hsa-miR-10a and miR-10b deviate only one nucleotide located at the center of their sequence and the primary hsa-miR-10b transcript may be equivalent to a promoter-associated RNA that could be targeted by miR-10a, suggesting the important role of miR-10a in breast cancer progression (19). Liu et al demonstrated that miR-18a prevented ER- $\alpha$ expression blocking the protective effects of estrogen and promoting the development of hepatocellular carcinoma (20). A previous study revealed that the expression of miR-18a-5p was enhanced in TNBC compared with luminal A (21). Aberrant upregulation of miR-18a could enhance autophagy in TNBC cells via inhibition of the mTOR signaling pathway (22) and decrease Dicer expression as well as increase paclitaxel resistance (23). Moreover, hsa-miR-135b was revealed to be upregulated in TNBC tissue which targeted estrogen receptor 1 (ESR1)-related proteins (24). Notably, miR-135b has been proposed as an oncogene involved in the pathogenesis of TNBC and the differential expression of miR-135b in blood could predict overall survival in follow-up of basal-like TNBC patients $(25,26)$. High expression of its family member, hsa-miR-135a, has been demonstrated to be associated with good prognosis in ER-positive tumors (27). Furthermore, hsa-miR-577 has been revealed to be dysregulated in several cancer types including gastric (28), bladder (29) and breast cancer (30). In addition, miR-577 has been demonstrated to suppress epithelial-mesenchymal transition and metastasis by inhibiting Rab25 expression in breast cancer (31). Our results demonstrated that hsa-miR-10a, hsa-miR-18a, hsa-miR-135b and hsa-miR-577 were significantly differentially expressed in the TNBC group and are potential candidate diagnostic markers of TNBC.

A 4-miRNA signature was identified after univariate and multivariate Cox's proportional hazards regression model analysis that was significantly correlated with the overall survival of TNBC patients. miR-148b, a tumor suppressor, has been reported to be dysregulated in pancreatic (32), non-small cell lung cancer (33) and hepatocellular carcinoma (34) through suppression of cell proliferation and invasion by targeting the AMPK $\alpha 1$ and WNT1/ $\beta$-catenin pathways. A previous study indicated that downregulation of miR-148b may be a molecular biomarker for the early detection of hepatocellular carcinoma and a prognostic marker (35). Increasing evidence indicates that miR-203 is involved in several cancers, including hepatocellular carcinoma (36), prostate (37), breast (38), gastric and colorectal cancers (39) through control of tumor cell proliferation, migration and invasive potential by interaction with target genes. Notably, dysregulated expression of miR-203 has been revealed to be associated with poorer survival of pancreatic tumors (40) and adenocarcinoma (41). As members of the miR-203 family, hsa-miR-203a and hsa-miR-203b may exert important roles in TNBC. Our results found 4 miRNAs (hsa-miR-148b, hsa-miR-203a, hsa-miR-203b and hsa-miR-3922) which may play a role in TNBC prognosis. 
To further explore the molecular mechanisms of the miRNA signature in TNBC, target genes of the miRNAs were predicted and functional annotation of targets was performed. The results of functional annotation of target genes revealed that regulation of anoikis, negative regulation of cellular amide metabolic process and protein insertion into mitochondrial membrane involved in the apoptotic signaling pathway were significant enriched GO terms. Anoikis is defined as apoptosis that is induced by inadequate or inappropriate cellular interaction with the extracellular matrix (42). Recently, more studies have confirmed that the breakdown of anoikis leads to the malignancy of mammary and colon cancers (43). Meanwhile, anoikis-resistance is a hallmark of metastasis (44). Therefore, these miRNAs may be involved in TNBC metastasis. According to pathway enrichment analysis of the miRNA signature targets, miRNAs in cancer, and the ErbB and prolactin signaling pathways were clearly enriched terms that encompassed most genes. Increasing evidence suggests that miRNAs participate in almost all aspects of cancer, including proliferation, apoptosis, angiogenesis and invasion/metastasis (45). Therefore, identification of clear diagnostic and prognostic miRNA biomarkers can contribute to cancer evaluation and treatment. Additionally, dimerization of ErbB receptors leads to induction of kinase activity that activates downstream MAPK and PI3K/AKT pathways which have significant involvement in tumor cell proliferation and survival (46). Notably, ErbB-2 overexpression has adverse prognostic value in breast cancer and thus, ErbB-directed strategies have been developed and used as treatments (47). The peptide hormone prolactin, synthesized by human breast cancer cells in culture, has been found to stimulate cell proliferation in an autocrine manner (48). Given the ability of prolactin to stimulate the proliferation of human breast cancer cells and the aberrant expression of its active receptors in breast carcinomas, it is fully consistent that prolactin plays a key role in breast cancer. Consequently, miRNAs in cancer, and the ErbB and prolactin signaling pathways may be significantly implicated in TNBC and inhibition of these pathways may be potential therapeutic strategies for TNBC patients.

However, there is also one limitation in the present study. The percentage of TNBC patients in breast cancer is so small that it is difficult to collect enough TNBC samples with follow-up information in a short time to verify the function of the identified miRNAs. Information of TNBC patients is now collected, however, just a few cases were obtained. The biological roles of these miRNAs in TNBC are still not clear and will be investigated in further experimental studies when enough TNBC samples are obtained.

In conclusion, the present study identified 4 aberrantly expressed miRNAs including hsa-miR-10a, hsa-miR-18a, hsa-miR-135b and hsa-miR-577 with diagnostic value for early diagnosis of TNBC patients, and subsequently, a 4-miRNA signature composed of hsa-miR-148b, hsa-miR-203a, hsa-miR-203b and hsa-miR-3922 was identified that may be a prognostic biomarker for predicting the overall survival of TNBC patients. However, further studies are required to validate these findings and the underlying molecular mechanisms of these miRNAs also require exploitation in combating TNBC in future.

\section{Acknowledgements}

We wish to express our warm thanks to Dr Lei Ma (Department of Breast Surgery, China-Japan Union Hospital of Jilin University, Changchun, China) who provided valuable guidance to our research.

\section{Funding}

No funding was received.

\section{Availability of data and materials}

All raw miRNA RNA-seq and clinical data of TNBC can be downloaded from TCGA data portal.

\section{Authors' contributions}

NL conceived and designed the study. CF analyzed the data and wrote the manuscript. Both authors read and approved the manuscript and agree to be accountable for all aspects of the research in ensuring that the accuracy or integrity of any part of the work are appropriately investigated and resolved.

\section{Ethics approval and consent to participate}

Not applicable.

\section{Patient consent for publication}

Not applicable.

\section{Competing interests}

The authors declare that they have no competing interests.

\section{References}

1. Torre LA, Bray F, Siegel RL, Ferlay J, Lortet-Tieulent J and Jemal A: Global cancer statistics, 2012. CA Cancer J Clin 65: $87-108,2015$

2. Chen W, Zheng R, Baade PD, Zhang S, Zeng H, Bray F, Jemal A, $\mathrm{Yu}$ XQ and He J: Cancer statistics in China, 2015. CA Cancer J Clin 66: 115-132, 2016.

3. Carey LA, Perou CM, Livasy CA, Dressler LG, Cowan D, Conway K, Karaca G, Troester MA, Tse CK, Edmiston S, et al: Race, breast cancer subtypes, and survival in the Carolina Breast Cancer Study. JAMA 295: 2492-2502, 2006.

4. Sorlie T, Tibshirani R, Parker J, Hastie T, Marron JS, Nobel A, Deng S, Johnsen H, Pesich R, Geisler S, et al: Repeated observation of breast tumor subtypes in independent gene expression data sets. Proc Natl Acad Sci USA 100: 8418-8423, 2003.

5. Croce CM and Calin GA: miRNAs, cancer, and stem cell division. Cell 122: 6-7, 2005.

6. Cascione L, Gasparini P, Lovat F, Carasi S, Pulvirenti A, Ferro A, Alder H, He G, Vecchione A, Croce CM, et al: Integrated microRNA and mRNA signatures associated with survival in triple negative breast cancer. PLoS One 8: e55910, 2013.

7. Gasparini P, Cascione L, Fassan M, Lovat F, Guler G, Balci S, Irkkan C, Morrison C, Croce CM, Shapiro CL, et al: microRNA expression profiling identifies a four microRNA signature as a novel diagnostic and prognostic biomarker in triple negative breast cancers. Oncotarget 5: 1174-1184, 2014.

8. Kleivi Sahlberg K, Bottai G, Naume B, Burwinkel B, Calin GA, Børresen-Dale AL and Santarpia L: A serum microRNA signature predicts tumor relapse and survival in triple-negative breast cancer patients. Clin Cancer Res 21: 1207-1214, 2015. 
9. Robinson MD, McCarthy DJ and Smyth GK: edgeR: A BioconductoR package for differential expression analysis of digital gene expression data. Bioinformatics 26: 139-140, 2010.

10. Yu G, Wang LG, Han Y and He QY: clusterProfiler: An R package for comparing biological themes among gene clusters. OMICS 16: 284-287, 2012.

11. Dawson SJ, Provenzano E and Caldas C: Triple negative breast cancers: Clinical and prognostic implications. Eur J Cancer 45 (Suppl 1): S27-S40, 2009.

12. Santarpia L, Qi Y, Stemke-Hale K, Wang B, Young EJ, Booser DJ, Holmes FA, O'Shaughnessy J, Hellerstedt B, Pippen J, et al: Mutation profiling identifies numerous rare drug targets and distinct mutation patterns in different clinical subtypes of breast cancers. Breast Cancer Res Treat 134: 333-343, 2012.

13. Zhang L, Huang J, Yang N, Greshock J, Megraw MS, Giannakakis A, Liang S, Naylor TL, Barchetti A, Ward MR, et al: microRNAs exhibit high frequency genomic alterations in human cancer. Proc Natl Acad Sci USA 103: 9136-9141, 2006.

14. Tan Y, Zhang B, Wu T, Skogerbø G, Zhu X, Guo X, He S and Chen R: Transcriptional inhibiton of Hoxd4 expression by miRNA-10a in human breast cancer cells. BMC Mol Biol 10: 12, 2009.

15. Yan Y, Wang Q, Yan XL, Zhang Y, Li W, Tang F, Li X and Yang P: miR-10a controls glioma migration and invasion through regulating epithelial-mesenchymal transition via EphA8. FEBS Lett 589: 756-765, 2015.

16. Markou A, Sourvinou I, Vorkas PA, Yousef GM and Lianidou E: Clinical evaluation of microRNA expression profiling in non small cell lung cancer. Lung Cancer 81: 388-396, 2013.

17. Gaur A, Jewell DA, Liang Y, Ridzon D, Moore JH, Chen C, Ambros VR and Israel MA: Characterization of microRNA expression levels and their biological correlates in human cancer cell lines. Cancer Res 67: 2456-2468, 2007.

18. Ma L: Role of miR-10b in breast cancer metastasis. Breast Cancer Res 12: 210, 2010.

19. Han J, Kim D and Morris KV: Promoter-associated RNA is required for RNA-directed transcriptional gene silencing in human cells. Proc Natl Acad Sci USA 104: 12422-12427, 2007.

20. Liu WH, Yeh SH, Lu CC, Yu SL, Chen HY, Lin CY, Chen DS and Chen PJ: MicroRNA-18a prevents estrogen receptor-alpha expression, promoting proliferation of hepatocellular carcinoma cells. Gastroenterology 136: 683-693, 2009.

21. Calvano Filho CM, Calvano-Mendes DC, Carvalho KC Maciel GA, Ricci MD, Torres AP, Filassi JR and Baracat EC: Triple-negative and luminal A breast tumors: Differential expression of miR-18a-5p, miR-17-5p, and miR-20a-5p. Tumour Biol 35: 7733-7741, 2014

22. Fan YX, Dai YZ, Wang XL, Ren YQ, Han JJ and Zhang H: MiR-18a upregulation enhances autophagy in triple negative cancer cells via inhibiting mTOR signaling pathway. Eur Rev Med Pharmacol Sci 20: 2194-2200, 2016.

23. Sha LY, Zhang Y, Wang W, Sui X, Liu SK, Wang T and Zhang H: MiR-18a upregulation decreases dicer expression and confers paclitaxel resistance in triple negative breast cancer. Eur Rev Med Pharmacol Sci 20: 2201-2208, 2016.

24. Dai X, Chen A and Bai Z: Integrative investigation on breast cancer in ER, PR and HER2-defined subgroups using mRNA and miRNA expression profiling. Sci Rep 4: 6566, 2014.

25. Uva P, Cossu-Rocca $P$, Loi F, Pira G, Murgia L, Orrù S, Floris M, Muroni MR, Sanges F, Carru C, et al: miRNA-135b contributes to triple negative breast cancer molecular heterogeneity: Different expression profile in basal-like versus non-basal-like phenotypes. Int J Med Sci 15: 536-548, 2018.

26. Paszek S, Gablo N, Barnas E, Szybka M, Morawiec J, Kołacińska A and Zawlik I: Dysregulation of microRNAs in triple-negative breast cancer. Ginekol Pol 88: 530-536, 2017.

27. Buffa FM, Camps C, Winchester L, Snell CE, Gee HE, Sheldon H, Taylor M, Harris AL and Ragoussis J: microRNA-associated progression pathways and potential therapeutic targets identified by integrated mRNA and microRNA expression profiling in breast cancer. Cancer Res 71: 5635-5645, 2011.

28. Pan HW, Li SC and Tsai KW: MicroRNA dysregulation in gastric cancer. Curr Pharm Des 19: 1273-1284, 2013.
29. Tatarano S, Chiyomaru T, Kawakami K, Enokida H, Yoshino H, Hidaka H, Yamasaki T, Kawahara K, Nishiyama K, Seki N, et al: miR-218 on the genomic loss region of chromosome 4p15.31 functions as a tumor suppressor in bladder cancer. Int J Oncol 39: 13-21, 2011.

30. Kolacinska A, Morawiec J, Fendler W, Malachowska B, Morawiec Z, Szemraj J, Pawlowska Z, Chowdhury D, Choi YE, Kubiak R, et al: Association of microRNAs and pathologic response to preoperative chemotherapy in triple negative breast cancer: Preliminary report. Mol Biol Rep 41: 2851-2857, 2014.

31. Yin C, Mou Q, Pan X, Zhang G, Li H and Sun Y: MiR-577 suppresses epithelial-mesenchymal transition and metastasis of breast cancer by targeting Rab25. Thorac Cancer 9: 472-479, 2018.

32. Zhao G, Zhang JG, Liu Y, Qin Q, Wang B, Tian K, Liu L, Li X, Niu Y, Deng SC, et al: miR-148b functions as a tumor suppressor in pancreatic cancer by targeting AMPKalpha1. Mol Cancer Ther 12: 83-93, 2013.

33. Yang JS, Li BJ, Lu HW, Chen Y, Lu C, Zhu RX, Liu SH, Yi QT, Li J and Song CH: Serum miR-152, miR-148a, miR-148b, and miR-21 as novel biomarkers in non-small cell lung cancer screening. Tumour Biol 36: 3035-3042, 2015.

34. Zhang JG, Shi Y, Hong DF, Song M, Huang D, Wang CY and Zhao G: MiR-148b suppresses cell proliferation and invasion in hepatocellular carcinoma by targeting WNT1/ $\beta$-catenin pathway. Sci Rep 5: 8087, 2015.

35. Ziari K, Zarea M, Gity M, Fayyaz AF, Yahaghi E, Darian EK and Hashemian AM: Downregulation of miR-148b as biomarker for early detection of hepatocellular carcinoma and may serve as a prognostic marker. Tumour Biol 37: 5765-5768, 2016.

36. Furuta M, Kozaki KI, Tanaka S, Arii S, Imoto I and Inazawa J: miR-124 and miR-203 are epigenetically silenced tumor-suppressive microRNAs in hepatocellular carcinoma. Carcinogenesis 31: 766-776, 2010.

37. Saini S, Majid S, Yamamura S, Tabatabai L, Suh SO, Shahryari V, Chen Y, Deng G, Tanaka Y and Dahiya R: Regulatory role of mir-203 in prostate cancer progression and metastasis. Clin Cancer Res 17: 5287-5298, 2011.

38. Zhang Z, Zhang B, Li W, Fu L, Fu L, Zhu Z and Dong JT: Epigenetic silencing of miR-203 upregulates SNAI2 and contributes to the invasiveness of malignant breast cancer cells. Genes Cancer 2: 782-791, 2011.

39. Chiang Y, Song Y, Wang Z, Chen Y, Yue Z, Xu H, Xing C and Liu Z: Aberrant expression of miR-203 and its clinical significance in gastric and colorectal cancers. J Gastrointest Surg 15: 63-70, 2011.

40. Greither T, Grochola LF, Udelnow A, Lautenschlager C, Wurl P and Taubert H: Elevated expression of microRNAs 155, 203, 210 and 222 in pancreatic tumors is associated with poorer survival. Int J Cancer 126: 73-80, 2010.

41. Hezova R, Kovarikova A, Srovnal J, Zemanova M, Harustiak T, Ehrmann J, Hajduch M, Svoboda M, Sachlova M and Slaby O: Diagnostic and prognostic potential of miR-21, miR-29c, miR-148 and miR-203 in adenocarcinoma and squamous cell carcinoma of esophagus. Diagn Pathol 10: 42, 2015.

42. Frisch SM and Francis H: Disruption of epithelial cell-matrix interactions induces apoptosis. J Cell Biol 124: 619-626, 1994.

43. Streuli $\mathrm{CH}$ and Gilmore AP: Adhesion-mediated signaling in the regulation of mammary epithelial cell survival. J Mammary Gland Biol Neoplasia 4: 183-191, 1999.

44. Park SH, Riley P IV and Frisch SM: Regulation of anoikis by deleted in breast cancer-1 (DBC1) through NF- $\mathrm{BB}$. Apoptosis 18: 949-962, 2013.

45. Garofalo M, Leva GD and Croce CM: MicroRNAs as anti-cancer therapy. Curr Pharm Des 20: 5328-5335, 2014.

46. Yarden Y and Sliwkowski MX: Untangling the ErbB signalling network. Nat Rev Mol Cell Biol 2: 127-137, 2001.

47. DiGiovanna MP, Stern DF, Edgerton SM, Whalen SG, Moore D II and Thor AD: Relationship of epidermal growth factor receptor expression to ErbB-2 signaling activity and prognosis in breast cancer patients. J Clin Oncol 23: 1152-1160, 2005.

48. Vonderhaar BK: Prolactin involvement in breast cancer. Endocr Relat Cancer 6: 389-404, 1999. 